\title{
The thrashed mass kinematic parameters establishment in the roller thrashing unit clearance
}

\author{
Azamat E. Bogus ${ }^{1, *}$, and Anastasia U. Pusikova ${ }^{1}$ \\ ${ }^{1}$ FSBEIHE Kuban State Agrarian University named after I.T.Trubilin, Krasnodar, Russia
}

\begin{abstract}
Planetary mechanisms are used to create the working movement of operating bodies of metallurgical and mining machines. Rolling working bodies, for the drive of which the planetary mechanisms are used are increasingly used in the designs of harvesters. The processes taking place in the differential threshing devices of harvesters are poorly studied due to the lack of information about the properties of the material in the case of rapidly changing stresses in them. The present work is a result of the analysis of theoretical and experimental researches in threshing. We described the technological scheme of the shock-vibration threshing device and the principle of its operation. There were studied the possible options of the placement of rollers and a concave. The relationship between the angular velocities of a roll, rollers and a concave was found out. The installation of three-sided rollers on the threshing device according to the scheme proposed by us makes it possible to shake the whole threshed rice mass from 75 to 110 times per second.
\end{abstract}

The principal scheme of the roller trashing unit with differential drive is presented in Figure 1. The rice mass that is held by the serving rolls 5 is received at transporter 4 constant speed $(\mathrm{Vn})$ in the working clearance between polyhedral rolls 2 and 3, drum and Concave rolling towards each other at equal relative angular speeds $\left(\omega_{2}=\omega_{3}\right)$. On the drum rolling at a frequency $\omega_{1}$ there are nine polyhedral rolls and six are in the concave. Arrangement angle of the drum rolls is twice as large as that of the concave.

In transition through the roller thrashing unit, the rice mass fulfills a complex motion. On the one hand, the rice mass is moved by the feeding rolls at transporting speed that is equal to serving speed, and on the other hand, it is subjected to consequent concave and drum rolls ribs strokes, that force it to fulfill relative oscillation movements in vertical plane $[1]$.

It is experimentally established, that in the working clearance the rice mass is in tense condition due to the fact that the rice mass serving speed is less than absolute circular drum and concave rollers speed $10 \div 15$ times.

\footnotetext{
*Corresponding author: azamat089@gmail.com
} 


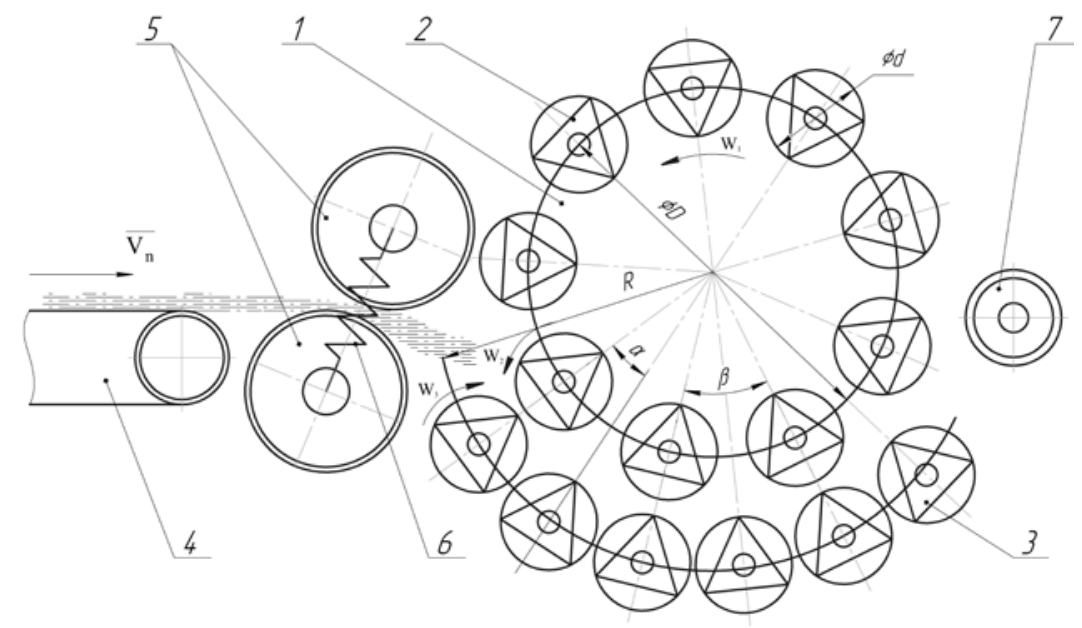

Fig. 1. The scheme of roller trashing unit operation.

To study the conformity of the rice mass movement we shall consider kinetics of the first pare of rollers at the moment when the drum and concave rollers axles are on the drum vertical radius (figure 2). The relative position of the first rollers pare is that under which the concave roller rib is perpendicular to drum radius and the concave roller rib is upward directed, the rib of the corresponding drum roller being directed towards the upper side of the concave roller.

Polyhedral rollers influence on the rice mass transportation character in the working zone of thrashing-separating unit is determined by the rollers geometry, by the way of their primary arrangement and by the correlation of rollers and drum rotation frequencies [2].

The study of the rice mass transportation regularity between the thrashing separating unit working zone rollers can be divided into two problems:

1. The establishment of regularity of the thrashed mass transportation under the influence of concave rollers.

2. The establishment of regularity of the thrashed mass transportation under the influence of concave rollers fulfilling complex movement.

We study the first problem that includes the threshed mass and concave rollers rotating on their own fixed axles interaction regularities examination.

Here two aspects could be outlined:

a) The establishment of the transportation regularity of the total thrashed mass layer when concave rollers ribs interact consequently and the rice mass is accepted as a deformed stem «pivot».

b) The regularity establishment of the fixed point movement on the thrashed mass during its transportation through the working thrasher unit clearance.

The study of the first aspect allows determination of the kinematic parameters the thrashed mass different points $D^{i}$ in the moment of their contact with the concave roller rib.

The solution of the second problem allows determination of the trajectory and kinematic parameters of the point $D_{0}^{\prime}$ fixed on a thrashed mass being in the thrasher unit working zone [3].

We shall consider the movement of the thrashed mass point $D^{i}$ when moving from the feeding rollers through first thrashing rollers pair working zone. The stem «pivot» fulfills 
the plane-parallel motion in the vertical plane at the portable progressive speed that is equal to the mass $V_{p}$ feeding speed and with the relative rotation movement around point $\mathrm{O}$.

In thrashed mass random point kinematic parameters determination and taking into account its the following assumptions are necessary and possible [4].

1. The thrashed mass uniformly fills the total capacity of the thrashing unit working zone $D F B E$.

2. The first co-stroke of the concave roller rib and the thrashed mass takes place when the side $C_{0} D_{0}$ is parallel to stem «pivot».

3. Under the roller rib co-stroke with the thrashed mass is deformed $D^{i}$, but is not detached from the roller rib as a result of the stroke interaction.

In accordance with the second assumption, the primary concave side position $D_{0} C_{0}$ is determined by the angle $\phi$ meaning depending on the sides number and the random roller rib position determined by the current angle $\alpha$ meaning. $A_{0} B_{0}$ - primary drum rollers position over the first concave roller, when its upper side is in position $D_{0} C_{0}$ [5].

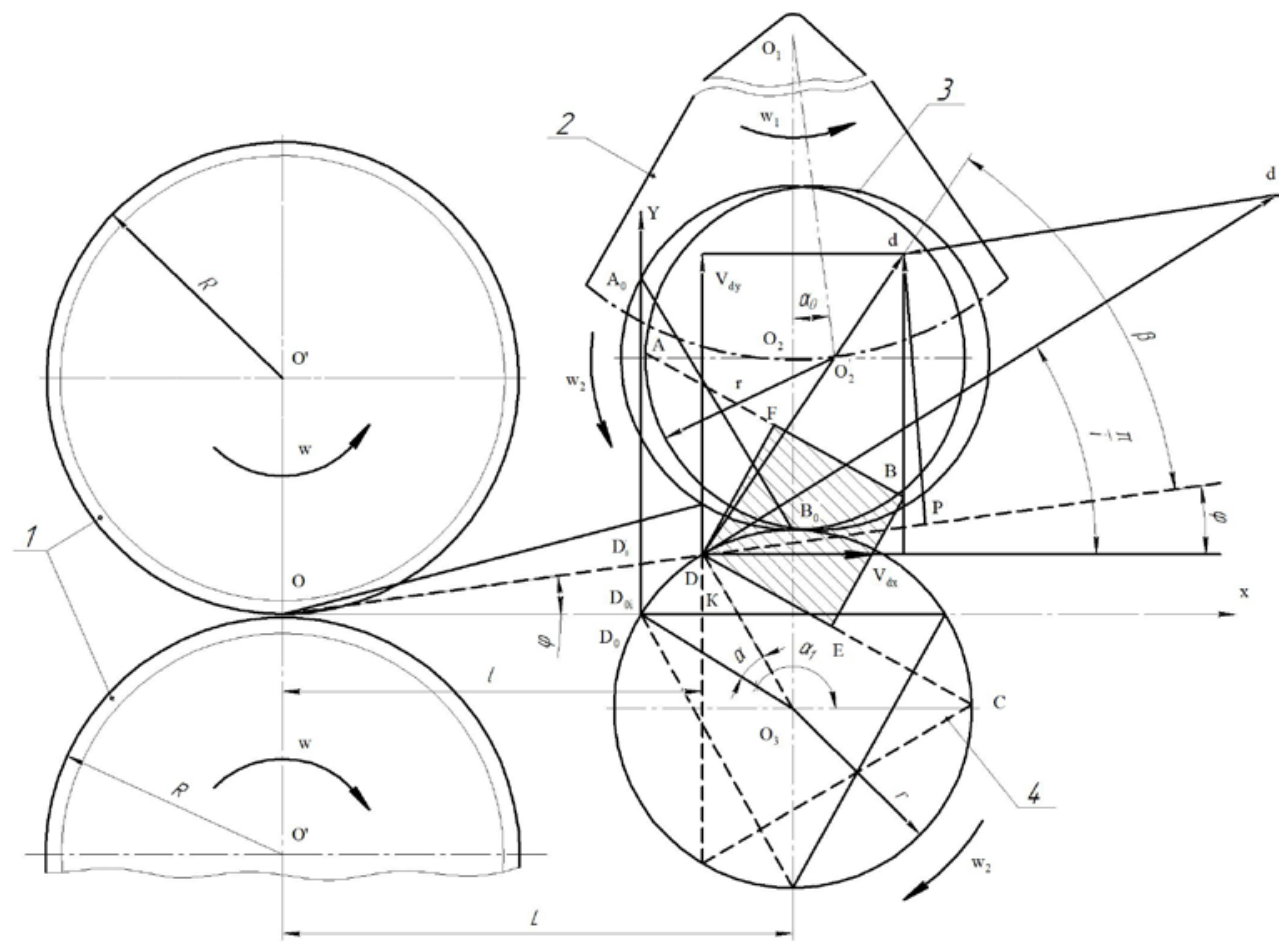

Fig 2. The scheme for the thrashed mass kinematic parameters determination. 1.-feeding rollers, 2drum, 3-drum roller, 4-concave drum.

In concave roller turn at angle $\alpha$ the side $D_{0} C_{0}$ takes the DC position and the rib $D^{i}$ random point.

The speed of rice mass feeding, in the absence of slip between the falling rollers is

$$
V_{p}=\omega R_{1}
$$


Where $\omega$ - the falling rollers relation speed $\mathrm{s}^{-1}$;

$R_{1}$ - the falling rollers radius, $\mathrm{m}$.

The circular speed of the concave roller is determined

$$
V_{o}=\omega_{2} r
$$

Where $\omega_{2}$-concave roller relation speed, $\mathrm{s}^{-1}$;

$r$ - circumcircle radius, $m$.

It is known that due to the friction force between the drum and concave rollers and thrashed mass, the latter is stretched towards the mass movement transmissible speed. This allows, with a negligible error, accept the moving rice mass that is between the falling rollers and the firs pair of polyhedral rollers as straight line $O D$ that is contiguous to the first concave roller rib in the point $D_{0}$. The straight line $O D$ fulfills oscillation movements around point 0 .

Portable (transmissible) speed of any thrashed rice mass point is directed towards the feeding speed and is determined by the expression (1).

The absolute speed $V_{D^{i}}$ of a random point at the moment of its costriking, with the rib $D$ is defined by the equation with the rib $D$ is defined by the equation [6].

$$
\begin{aligned}
& \bar{V}_{D^{i}}=\bar{V}_{O}+\bar{V}_{D^{i} O} \\
& \bar{V}_{D^{i}}=\bar{V}_{D}+\bar{V}_{D^{i} D}
\end{aligned}
$$

Where $\bar{V}_{D^{i} O}$-the relative rotation speed vector of the rice mass point $D^{i}$ relative to point $\mathrm{O}$.

$\bar{V}_{D^{i} D}$ - the relative movement speed vector of the thrashed mass point $D^{i}$ relative to concave roller rib point $D$.

The speed $\bar{V}_{D^{i} O}$ is perpendicular $O D$ and the vector $\bar{V}_{D^{i} D}$ is parallel to $O D$.

We take point $D$ as a pole and produce speeds plan for random point of the thrashed mass $D^{i}$ using equations (3) and (4),

In the speeds plan me have.

$\overline{D d^{\prime}}$ - absolute speed vector of the rice mass point $D^{i}$;

$\overline{D d}$ - Linear speed vector of the roller rib point $D$;

$\overline{D n}$ - portable (transmissible) speed vector of the rice mass random point $D^{i}$ - equal to portable (transmissible)speed vector of the straw «pivot» point (equal to the thrashed mass feeding speed- $V_{p}$ )

$\overline{d^{\prime} d}$ - relative speed vector of the thrashed mass point $D^{i}$ relative to concave roller rib (point $D$ ).

$\overline{d^{\prime} n}$ - relative speed vector of the thrashed mass point $D^{i}$ relative to point $\mathrm{O}$ in rotation movement of straw «pivot»around point $\mathrm{O}$.

The speeds plan is not designed in scale as the portable (transmissible) speed module of the thrashed mass random point $V_{D n}$ is considerably less than the point $D$ linear speed 
module of the roller rib $V_{D d}$ and in scale the real portable (transmissible) speed within a draft would have been too insignificant and that would have brought about errors in the immediate speed plan utilization in scale.

To define the point $D^{i}$ speed of the thrashed mass $V d_{n}^{\prime}$ relative to point $\mathrm{O}$ - stem «pivot» compression it is necessary to have the meaning of the thrashed mass relation frequency around this point [7].

$$
V_{D_{n}^{\prime}}=\omega_{c} D O
$$

Where rotation speed

$$
\omega_{c}=\omega_{2} \frac{d \phi}{d \alpha} .
$$

We express the stem «pivot» turning angle $\phi$ around point 0 trough current meaning of concave roller turning angle- $\alpha$

$$
\operatorname{tg} \phi=\frac{D K}{K O}=\frac{D K}{l}=\frac{r\left[\cos \left(\frac{\pi}{i}-\alpha\right)-\cos \frac{\pi}{i}\right]}{L-r \sin \left(\frac{\pi}{i}-\alpha\right)}
$$

where $r$ - roller circumcircle radius;

$i$ - roller sides number;

$L$ - distance between feeding rollers rotation axles and the concave first roller.

The stem «pivot» turning angle $\phi$ has the maximum meaning when $\alpha=\frac{\pi}{i}$; (by any roller sides number). The more is the $\phi$ angle meaning, the less is the roller sides number. In our researches the number of the roller sides changed within $3 \div 6$, and that means that the maximum $\varphi$ angle meaning and, consequently, the maximum deviation angle of the rice mass will be under $i=3$. It should be noted that the maximum rice mass deviation angle coincides with the upper most roller rib position [8].

In analyzing expression (7) we found that the maximum deviation angle $\varphi$ meaning corresponds to the following meaning of angle $\alpha$

$$
\operatorname{tg} \alpha=\frac{r\left(1-\cos \frac{\pi}{i}\right)}{L} .
$$

In a real thrasher $R_{1}=2 r, L=5 r$; hence the maximum deviation angle meaning of the stem «pivot» from horizontal line is $\phi_{\max } \cong 6^{0}$,that is why we can approximately accept $\operatorname{tg} \phi \cong \phi$.

Therefore, 


$$
\phi=\frac{r\left[\cos \left(\frac{\pi}{i}-\alpha\right)-\cos \frac{\pi}{i}\right]}{L-r \sin \left(\frac{\pi}{i}-\alpha\right)}
$$

We shall differentiate expression according to $\alpha$ and having fulfilled some transformations we have

$$
\frac{d \phi}{d \alpha}=\frac{r L \sin \left(\frac{\pi}{i}-\alpha\right)-r^{2}\left[1-\cos \left(\frac{\pi}{i}-\alpha\right) \cos \frac{\pi}{i}\right]}{\left[L-r \sin \left(\frac{\pi}{i}-\alpha\right)\right]^{2}}
$$

Substituting consecutively expression (10) into (6) we get

$$
\omega_{C}=\omega_{2} \cdot \frac{r L \sin \left(\frac{\pi}{i}-\alpha\right)-r^{2}\left[1-\cos \left(\frac{\pi}{i}-\alpha\right) \cos \frac{\pi}{i}\right]}{\left[L-r \sin \left(\frac{\pi}{i}-\alpha\right)\right]^{2}}
$$

Substituting the stem «pivot» angular speed meaning $\omega_{C}$ into expression we get

$$
V_{D^{\prime} O}=\omega_{2} \cdot \frac{r L \sin \left(\frac{\pi}{i}-\alpha\right)-r_{2}^{2}\left[1-\cos \left(\frac{\pi}{i}-\alpha\right) \cos \frac{\pi}{i}\right]}{\left[L-r \sin \left(\frac{\pi}{i}-\alpha\right)\right]^{2}} \cdot O D
$$

We simplify expression (12) equalying $O D \cong l$, that is quite possible and we get current meaning $O D \approx l=L-r \sin \left(\frac{\pi}{i}-\alpha\right)$ for small angle $\phi$ meanings.

Substituting current meaning $O D$ into the equation (12) we get

$$
V_{D^{\prime} O}=\omega_{2} \cdot \frac{r L \sin \left(\frac{\pi}{i}-\alpha\right)-r^{2}\left[1-\cos \left(\frac{\pi}{i}-\alpha\right) \cos \frac{\pi}{i}\right]}{L-r \sin \left(\frac{\pi}{i}-\alpha\right)}
$$

The relative movement speed of the thrashed mass random point $D^{i}$ relative to roller rib $D\left(V_{d^{\prime} d}\right)$ is the speed at which the thrashed mass is carded by the concave roller rib [9]. 
We find the carding speed meaning in speeds plan (fig 2).

$$
V_{d^{\prime} d}=V_{o h}=V_{D^{i} d} \cdot \cos \left[\left(\frac{\pi}{i}-\alpha\right)-\phi\right]-V_{p},
$$

or

$$
V_{d^{\prime} d}=\omega_{2} r \cos \left[\left(\frac{\pi}{i}-\alpha\right)-\phi\right]-\omega R
$$

As the meaning of $\phi$ is small we suppose that $\cos \phi \approx 1$ and substituting stem «pivot» turning angle meaning $\phi$ out of (9) into (14) after some transformations we get

$$
V_{d^{\prime} d}=\omega_{2} r \cdot \frac{L \cos \left(\frac{\pi}{i}-\alpha\right)-r \sin \left(\frac{\pi}{i}-\alpha\right) \cos \frac{\pi}{i}}{L-r \sin \left(\frac{\pi}{i}-\alpha\right)}-\omega R
$$

The carding concave rollers speed was determined out of expression (15) under the following primary conditions: $\omega=10 \mathrm{c}^{-1} ; \omega_{2}=200 \mathrm{c}^{-1}, r=0,06 \mathrm{M}, L=0,5 \mathrm{M}, R=0,1 \mathrm{M}$. The graph dependencies $V_{d^{\prime} d}=f(\alpha, i)$ were obtained (fig. 3)

Table1

\begin{tabular}{|c|c|}
\hline Sides number & Angle $\alpha$ change range \\
\hline 3 & $0 \leq \alpha \leq \frac{2}{3} \pi$ \\
\hline 4 & $0 \leq \alpha \leq \frac{\pi}{2}$ \\
\hline 5 & $0 \leq \alpha \leq \frac{2}{5} \pi$ \\
\hline 6 & $0 \leq \alpha \leq \frac{\pi}{3}$ \\
\hline 7 & $0 \leq \alpha \leq \frac{2,11}{7,5} \pi$ \\
\hline 8 & $0 \leq \alpha \leq \frac{\pi}{4}$ \\
\hline
\end{tabular}




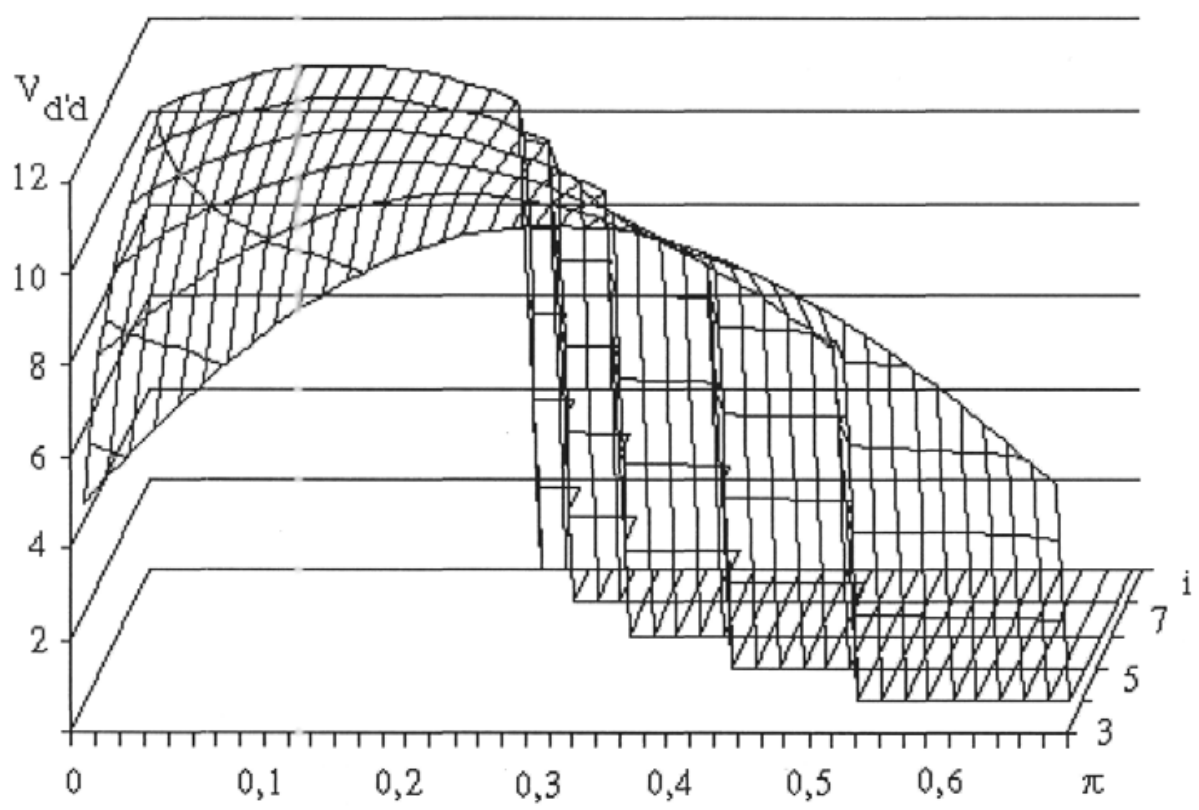

Fig. 3. The surface of rice mass carding speed dependencies response in function $V=f(\alpha, i)$

In the functional dependence (15) research is the range of variable parameters functions change, the obtained response surface demonstrates harmonious function and its periods are inversely proportional to the number of rollers sides and the amplitude is directly proportional to them.

We find the absolute speed $V_{d^{\prime}}$ of the thrashed rice mass random point $D^{i}$ out of speeds plan

$$
V_{d^{\prime}}=\frac{V_{p}}{\cos \beta}=\frac{\omega R}{\cos \beta}
$$

where $\beta$ - the angle between the speeds $V_{p}$ and $V_{d}$, vectors.

The angle $\beta$ meaning between the speeds $V_{p}$ and $V_{d}$, vectors equals.

\section{Conclusions}

The equations $(15,16)$ defining kinematic parameters of moving rice mass random point $D^{i}$ in costriking with concave roller rib were oblaired, in the interval between the falling rollers and the concave first working pair of rollers in accordance with constructive, kinematic and technology parameters of thrashing-separativy unit operation:

-the thrashed mass random point relative speed meaning relative to concave roller rib point $D$, equal to concave rollers carding speed (15).

-the thrashed mass random point absolute speed meaning (16). 


\section{References}

1. Papusha S.K., Belousov S.V., Bogus A.E., Konovalov V.I. Theoretical studies of tobacco stalk interaction with a leaf-separating unit // International Journal of Applied Engineering Research. 2016. V. 11. № 8. p. 5610-5613.

2. Bogus S. N. Differential thrashing device power estimation. Mechanization of agricultural production. - Works of Kuban State Agricultural Institute, 1978, part 156 (184), p. 47-53.

3. Tselikov A. I., Ritman P. I. Planetary mills roller effort definition. - In the collection of Works: Rolling mills and rolling technology. - M.; Moscow Higher Technical college, 1958, № 84, p. 5-30.

4. Skorodumov A. N. On the theory of planetary rolling. - Higher Educational Institutions News. Ferrous Metal Industry, 1959, № 2, p. 62-71.

5. Vashchenko Y. F. Planetary thrashing device for wheat. - Scientific report of agricultural machines department. Kuban State Agricultural Institute, 1969. Krasnodar, 1970, p. 24.

6. Gudkov A. N., Zadneprovsky R. P. On the substantiation of the operating parts parameters for vibration thrashing. Mechanization and Electrification of Socialist Agriculture, 1964 № 1, p.21-24.

7. Bogus A.E., Grachev E.A. A study of a shock pulse of a roller rib of the planetary threshing device on grain mass // Polythematic network electronic scientific journal of Kuban State Agrarian University- Krasnodar: Printing house «KubSAU». 2018. № 135., p. 188-199.

8. Isaev Yu. M and Semashkin N. M, Critical Frequency of Rotation in the Spiral Screw De-vice, International Journal of Mechanical Engineering and Technology, 9(5), 2018, pp. 541-547.

9. Vineet Kumar Bhagat, Anil Kumar Prasad and Arvind Kumar Lai Srivastava, Physical and Mechanical Performance of Luffa-Coir Fiber Reinforced Epoxy Resin Based Hybrid Compo-sites. International Journal of Civil Engineering and Technology, 8(6), 2017, pp. 722-731. 